\title{
Preditores Psicossociais de Sintomas Osteomusculares: A Importância das Relações de Mediação e Moderação
}

\author{
Psychosocial Predictors of Musculoskeletal Symptoms: \\ The Relevance of Mediated and Moderated Relationships
}

\author{
Fernanda Amaral Pinheiro*, Bartholomeu Tôrres Tróccoli, \& Maria das Graças Torres da Paz \\ Universidade de Brasília
}

\begin{abstract}
Resumo
Este trabalho teve como objetivo o desenvolvimento de um modelo teórico que associa variáveis de natureza psicossocial, presentes no ambiente de trabalho, a sintomas de distúrbios osteomusculares, investigando possíveis relações de mediação e moderação. Uma amostra de 494 trabalhadores de um banco estatal preencheu um questionário que incluiu medidas dos aspectos psicossociais do ambiente de trabalho, reatividade ao estresse (exaustão emocional), estilos de coping e uma escala de freqüência de sintomas osteomusculares, além de questões sobre hábitos e estilo de vida. Os resultados mostraram que exaustão emocional foi o mais poderoso preditor de relato de sintomas, o que é um indício de que a reação ao estresse seja uma via pela qual os fatores psicossociais afetam a saúde osteomuscular.

Palavras-chave: Distúrbios osteomusculares; fatores psicossociais; exaustão emocional; mediadores; moderadores.
\end{abstract}

\begin{abstract}
This study aimed to develop a theoretical model that associates psychosocial variables in occupational settings to musculoskeletal symptoms, looking for possible relationships of mediation and moderation. A sample of 494 government workers of a bank (owned and administered by the government) responded to a questionnaire that included psychosocial variable measurements of the occupational setting, stress reactivity (emotional exhaustion), coping styles and a scale of musculoskeletal symptoms frequency, besides questions on habits and lifestyle. The results showed that emotional exhaustion was the most powerful predictor of symptoms report, which indicates the stress reaction is a path by which psychosocial aspects may affect musculoskeletal health. Keywords: Musculoskeletal disturbances; psychosocial factors; emotional exhaustion; mediators; moderators.
\end{abstract}

Na área de saúde ocupacional, os distúrbios osteomusculares têm se destacado como uma das patologias do trabalho mais freqüentemente citadas, ao lado da surdez ocupacional, as dermatoses, as doenças pulmonares e a intoxicação por benzeno (Miranda, 1998).

Entenderemos por distúrbios osteomusculares relacionados ao trabalho (DORT) um conjunto de doenças que se caracterizam pela ocorrência de sintomas tais como dor, parestesias (dormência, formigamento, diminuição da sensibilidade), sensação de peso e/ou fadiga, que acometem principalmente os membros superiores (Sato et al., 1993).

A Organização Mundial de Saúde (OMS) caracterizou as doenças relacionadas ao trabalho como multifatoriais, o que implica em sua múltipla causalidade, incluindo fatores de ordem física, organizacional, psicossocial, individual e sociocultural (Bernard, 1997; Rio, 1998). Entretanto, o modelo biomédico tradicional encara com certo ceticismo a contribuição dos fatores psicossociais, chegando mesmo a propor o seu abandono devido ao "componente fortemente abstrato e intuitivo" que, “... a longo prazo pode desacreditar as DORT como um todo” (Rio, 1998, p.109).
De fato, os estudos originados sob a ótica psicossocial nem sempre têm contribuído para o esclarecimento da etiologia dos DORT, particularmente quando atribuem a estes causas exclusivamente psicológicas, caracterizando-se pelo reducionismo e pela ausência de evidências empíricas que os sustentem.

Fator, demanda ou aspecto psicossocial são denominações que incluem variáveis de diferentes dimensões. Para Toomingas, Theorell, Michelsen, e Nordemar (1997), os fatores psicossociais ocupacionais descrevem como a organização do trabalho é percebida pelos trabalhadores e administradores. O International Labour Office (ILO) os definiu como a interação entre os aspectos do ambiente de trabalho (ou externos a ele) e características do indivíduo, que afetam o bem-estar e o desempenho, com ênfase nos efeitos psicológicos.

Em termos práticos, os estudos denominam psicossocial toda variável de natureza não-física, relacionada a efeitos sobre a saúde e/ou ao desempenho, muitas vezes tratando-as como estressores ocupacionais (Bergqvist, Wolgast, Nilsson, \& Voos, 1995a; Bernard, 1997; Bongers, de Winter, Kompier, \& Hildebrandt, 1993; Sauter, Schleifer, \& Knutson, 1991). Neste estudo, o termo fatores psicossociais é usado de forma a incluir,
"Endereço: Integra Psicologia e Pesquisa Aplicada, SGAN 914
Cj.H, Bl. A, sl.215, Asa Norte, 70790 140, Brasilia, DF fernanda@integrapesquisa.com.br 
especificamente, a percepção dos trabalhadores quanto à sobrecarga, suporte material ao desempenho, suporte social do grupo, gestão organizacional do desempenho e à política de remuneração (pagamento e desenvolvimento). Desta forma, busca-se delimitar os fatores psicossociais na esfera do ambiente ocupacional, abordando-os em sua dimensão relacional para com a organização, como um todo, o grupo de colegas e a chefia imediata.

Discute-se, atualmente, se os fatores psicossociais estariam, de fato, na origem dos sintomas osteomusculares ou se a relação entre estes e sintomas seria mediada por carga física, sendo esta, na verdade, o fator etiológico de primeira ordem.

Bernard (1997), na extensa revisão de literatura realizada pelo National Institute for Occupational Safety and Health (NIOSH), afirma que muitos estudos têm encontrado associações positivas, embora modestas, entre os fatores psicossociais e os distúrbios osteomusculares (particularmente nas regiões do pescoço, ombros, extremidades superiores e região dorsal), mesmo após o ajuste da variável carga física.

Um refinamento do modelo etiológico dos DORT foi proposto por Toomingas et al. (1997). Aqueles autores postularam que carga física seria associada a sintomas e sinais em tendões, articulações e nervos (regiões anatômicas periféricas), enquanto que os sintomas e sinais associados à exposição às condições psicossociais seriam localizados nas regiões anatômicas centrais, incluindo pescoço, ombros e dorso. Outros estudos também identificaram a especificidade da relação entre os fatores psicossociais e a ocorrência de sintomas na região de pescoço e ombros (Bernard, Sauter, Fine, Petersen, \& Hales, 1994; Houtman, Bongers, Smulders, \& Kompier, 1994; Leino \& Haenninen, 1995).

Há evidências de que as condições adversas de natureza psicossocial no trabalho relacionam-se positivamente à ocorrência de sintomas osteomusculares em regiões anatômicas específicas, independentemente da carga física associada à atividade. Entretanto, a magnitude da relação é pequena e variável entre os estudos.

\section{Vias de Associação}

A partir da revisão da literatura realizada, conclui-se que a associação entre fatores psicossociais e DORT poderia se dar por meio de uma das seguintes vias (Bernard, 1997; Bongers et al., 1993; Theorell, 1996):

- Os fatores psicossociais afetariam diretamente a carga física, como a pressão do tempo aumenta a ocorrência da aceleração dos movimentos e da postura inadequada;

- Os fatores psicossociais, juntamente às estratégias de coping ou enfrentamento disfuncionais, podem levar ao estresse e resultar em um aumento da contração muscular que, a longo prazo, poderia levar ao desenvolvimento ou exacerbação de sintomas; e,

- Fatores psicossociais poderiam influenciar a sensibilidade à dor, baixando o limiar de percepção, o que resultaria no aumento da freqüência do relato de sintomas.
Elovainio e Sinervo (1997) testaram dois modelos de associação entre fatores psicossociais e sintomas osteomusculares: o modelo da mediação de carga física e o modelo que associa fatores psicossociais e sintomas de estresse. Os resultados, utilizando análise de equação estrutural (LISREL), evidenciaram melhores índices de adaptabilidade do segundo modelo aos dados. Há, portanto, indícios da intermediação do estresse na relação entre o ambiente psicossocial e a saúde osteomuscular.

\section{A Contribuição da Teoria do Estresse Ocupacional}

Selye (1985) propôs que o estresse é um processo biológico por meio do qual o organismo tenta se adaptar a desafios do ambiente, por meio de respostas de enfrentamento em busca da sobrevivência. Smith e Carayon (1996), reinterpretando os pressupostos de Selye, sugeriram que as reações fisiológicas subjacentes ao processo de estresse teriam o potencial de aumentar a suscetibilidade do organismo como um todo à doença e de tecidos específicos a traumas cumulativos, principalmente considerando que tensão e fadiga muscular, próprios da fase de exaustão do processo, segundo Selye, são também os primeiros sintomas de distúrbios osteomusculares.

Em seu estudo, já citado, Toomingas et al. (1997) sugeriram que a tensão muscular induzida pelo estresse ou a falta de habilidade para relaxar poderiam mediar condições psicossociais precárias e predizer dor muscular.

Tensão muscular decorrente do estresse pode ser um dos mecanismos que associam o estresse a sintomas osteomusculares. Utilizando registro eletromiográfico como medida de morbidade, Westgaard (1996) relatou que atividade muscular involuntária poderia resultar de fatores motivacionais comuns na vida no trabalho e que tais mecanismos poderiam explicar dor osteomuscular, fibromialgia e mialgia do trapézio. Aquele autor apresentou evidências de que o tônus muscular contínuo, ainda que de baixa intensidade está associado à ativação das unidades motoras de baixo limiar, pelo menos no caso da mialgia do trapézio.

Outra via de associação entre o estresse e o desenvolvimento de distúrbios osteomusculares sugerido por Smith e Carayon (1996) seria a das estratégias de coping, ou formas de enfrentamento do estresse. Lima (1997) sugeriu que a auto-aceleração no desempenho da atividade, comum entre indivíduos acometidos de DORT/LER, seria uma forma de tentar reassumir algum controle sobre o ritmo da tarefa, especialmente quando a organização do trabalho permite pouco ou nenhum outro tipo de controle por parte do trabalhador. Este também parece ser o ponto de vista de Smith e Carayon (1996), que consideraram que tarefas pobres em significado e pouco desafiadoras do ponto de vista cognitivo podem levar o trabalhador a se engajar em métodos de trabalho impróprios em resposta às frustrações do ambiente.

A hipótese de uma relação entre o estresse, fatores psicossociais do ambiente de trabalho e sintomas osteomusculares é, portanto, bastante plausível e já encontra 
algum respaldo empírico. É preciso investigar se o estresse é um fator que aumenta a probabilidade de ocorrência do efeito - um fator mediador - ou se ele é condição necessária para a sua verificação - um fator moderador. É necessário ainda que os estudos confiram validade de construto às medidas dos fatores psicossociais, dada a estreita relação que existe entre estes e medidas de reatividade ao estresse ocupacional.

Discussões desta natureza ressaltam a importância de se ampliar o estudo sobre os antecedentes psicossociais dos DORT de forma a incluir a exploração de relações indiretas e mediadoras nos modelos preditivos, o que possibilitaria a investigação de associações que reproduzem mais fielmente a complexidade do cenário real.

\section{A Contribuição Deste Estudo: Objetivo, Variáveis e Hipóteses}

$\mathrm{O}$ objetivo deste trabalho foi verificar empiricamente a adequação do modelo de relações que associa variáveis do ambiente ocupacional a sintomas osteomusculares, considerando relações diretas e indiretas. Neste modelo, as variáveis do ambiente ocupacional são potenciais fatores de risco para sintomas osteomusculares: a) demandas psicossociais: percepção de sobrecarga, do estilo de gestão da chefia, da gestão organizacional do desempenho, de suporte material, de suporte social do grupo e da política de pagamento e desenvolvimento da empresa; e, b) carga física decorrente do tipo de atividade realizada.

Sintomas osteomusculares constituem o conjunto de variáveis dependentes do estudo. Para fins de análise e apresentação dos resultados, as regiões anatômicas foram divididas em centrais (cervical, ombros, dorsal e lombar) ou periféricas (braços, antebraços, cotovelos, punhos/mãos/ dedos).

Um terceiro conjunto de variáveis foi incluído no modelo. Supõe-se que estas atuem como mediadores ou moderadores da relação entre o ambiente ocupacional e sintomas. São elas: a) variáveis demográficas: idade, gênero; b) variáveis relativas a hábitos e estilo de vida: tabagismo, prática de exercícios físicos e carga física de natureza não ocupacional; e, c) variáveis disposicionais: estilos de coping e exaustão emocional.

$\mathrm{O}$ modelo sugere que fatores relativos ao ambiente organizacional encontram-se presentes na etiologia dos distúrbios osteomusculares por duas vias independentes: a da carga física e a via das demandas psicossociais. Carga física pode ser moderada ou mediada por variáveis demográficas, hábitos, variáveis disposicionais e por exaustão emocional, ou pode ter efeito direto na saúde osteomuscular, acarretando sintomas em regiões anatômicas periféricas. A via das demandas psicossociais seria mediada ou moderada por fatores demográficos, hábitos, variáveis disposicionais e por exaustão emocional, e estaria associada à ocorrência de sintomas nas regiões anatômicas centrais.

\section{Método}

\section{Participantes}

Os dados foram coletados entre julho e agosto de 2000, em uma amostra randômica de 1800 bancários, trabalhadores em um banco estatal, em Brasília. Os participantes desenvolviam atividades diversas nas áreas de escritório e atendimento ao público. Por meio do malote interno da empresa, estes receberam o instrumento juntamente a uma carta explicando o objetivo da pesquisa e garantindo o sigilo das informações. Embora 508 questionários houvessem retornado preenchidos (28\%), 14 foram excluídos da análise por apresentarem dados faltosos. A taxa de respostas obtida foi considerada compatível com o esperado em pesquisas de resposta espontânea, naquela organização, e não houve controle dos não-respondentes. As análises estatísticas foram baseadas em 494 respondentes, dos quais cinqüenta por cento eram homens. A idade variou entre 31 a 55 anos $(m=39,7 ; d p=5,5)$. O tempo médio de permanência no serviço foi de 15,7 anos $(d p=5,4)$. Setenta e cinco por cento dos respondentes eram casados ou viviam maritalmente. De todos, $73 \%$ haviam completado um curso de nível superior. Da amostra, $55 \%$ constituíam-se de empregados lotados na matriz, enquanto que $15 \%$ estavam em sede de filial e $30 \%$ eram lotados em agências.

\section{Medidas}

Variáveis demográficas e relativas a hábitos/estilo de vida: Foram mensuradas como segue: $i d a d e$ (em anos); gênero ( $1=$ mulheres; $2=$ homens); estado civil ( $1=$ casado/vive maritalmente; $2=$ solteiro/vive só); tabagismo ( $1=\mathrm{sim}$; $2=$ não); prática de exercícios físicos $(1=\operatorname{sim} ; 2=$ não).

Carga física: Foi investigada a carga física de natureza ocupacional e não-ocupacional. Carga física ocupacional foi traduzida como a freqüência de digitação, e mensurada pelas variáveis tipo de atividade lotação. Para medir tipo de atividade, um especialista da área de cargos e salários da organização codificou as funções relatadas pelos empregados em uma escala de cinco pontos de freqüência de digitação. Posteriormente, a variável foi recodificada para 3 pontos (1=baixa digitação; $2=$ média digitação; $3=$ alta digitação), para facilitar a interpretação dos resultados. Lotação foi medida como segue: $1=$ subsistema matriz; $2=$ subsistema filial; $3=$ subsistema agência, assumindo-se que haveria diferentes padrões de freqüência de digitação para a lotação em cada subsistema. A variável foi posteriormente dicotomizada, assumindo os valores $\mathrm{O}=$ não filial e $1=$ filial, para facilitar a visualização do contraste nas análises de regressão logística.

Carga física não-ocupacional: Foi mensurada por três variáveis dummy: exercício de atividades domésticas, cuidar de crianças em idade pré-escolar e uso do microcomputador doméstico $(\mathrm{O}=$ atividade não faz parte do dia a dia; $1=$ atividade faz parte do dia a dia).

Demandas Psicossociais: Foram mensuradas por seis subescalas que incluíram Sobrecarga, 8 itens, coeficiente alpha=0,85; Suporte Material, 9 itens, coeficiente 
alpha=0,77; Percepção do Estilo de Gestão da Chefia, 13 itens, coeficiente alpha=0,93, Suporte social do grupo, 3 itens, coeficiente alpha=0,79; Gestão Organizacional do Desempenho, 5 itens, coeficiente alpha $=0,77$ e Percepção da Política de Pagamento e Desenvolvimento, 4 itens, coeficiente alpha=0,79. Os seis fatores explicaram 49,6\% da variância total (Pinheiro \& Tróccoli, 2001).

Exaustão Emocional: Medida da variável exaustão emocional utilizada neste estudo foi desenvolvida por Tamayo e Tróccoli (2000). O instrumento é composto de 11 itens que se dividem em duas subescalas, a saber, exaustão psicológica (4 itens) e percepção de desgaste ( 7 itens), com índices de confiabilidade alpha igual a 0,89 e 0,91 , respectivamente. Optou-se pela utilização da subescala exaustão psicológica, e não a escala geral, em razão da forte correlação encontrada entre sobrecarga e percepção de desgaste $(r=0,54 ; p<0,01)$. A estrutura fatorial foi confirmada na amostra do estudo final, com os mesmos itens e índices de confiabilidade.

Coping: Utilizou-se a tradução para a língua portuguesa da escala desenvolvida por Latack (1986) para a mensuração de coping no ambiente de trabalho. A escala é composta de 46 itens cobrindo três dimensões de coping ocupacional: esquiva, controle e manejo de sintomas. Os índices de confiabilidade da versão brasileira foram considerados satisfatórios: (coeficiente alpha $=0,77 ; 0,79 ; 0,81$, respectivamente). A investigação da estrutura fatorial da escala nesta amostra replicou aqueles achados, com índices de confiabilidade ligeiramente superiores, a saber: esquiva, 10 itens, coeficiente alpha $=0,80$; controle, 16 itens, coeficiente alpha $=0,82$ e manejo de sintomas, 10 itens, coeficiente alpha $=0,83$. Todos estes instrumentos foram respondidos em uma escala de freqüência de cinco pontos ( $1=$ Nunca; $2=$ Raramente; $3=$ Às vezes; $4=$ Freqüentemente; $5=$ Sempre).

Sintomas osteomusculares: Foram registrados a partir de uma versão reduzida do Nordic Musculoskeletal Questionnaire (NMQ) (Kuorinka et al., 1987), na qual os respondentes são convidados a registrar, em uma escala de 4 pontos, a freqüência em que sentiram dor ou desconforto nos últimos 12 meses em nove regiões anatômicas (pescoço, ombros, braços, cotovelos, antebraços, punhos/mãos/dedos, dorso, região lombar e extremidades inferiores). A validade concorrente da escala traduzida com a história clínica foi de 0,86 (Pinheiro, Tróccoli, \& Carvalho, 2001). A análise fatorial do instrumento permitiu que as respostas dos sujeitos fossem agrupadas em dois fatores: região anatômica central e periférica. Estas variáveis foram posteriormente dicotomizadas ( $1=$ nunca ou raramente apresenta sintomas; $2=$ com freqüência ou sempre apresenta sintomas), para permitir a utilização da técnica de análise de regressão logística.

\section{Análise dos Dados}

Estatísticas descritivas foram calculadas para a descrição da amostra. Teste te ANOVA foram utilizados para comparar médias e índices de correlação foram usados para investigar a relação entre as variáveis do estudo. Excetuando-se as variáveis demográficas, de hábitos/estilo de vida e o relato de sintomas, as demais foram tratadas como variáveis contínuas.

Análises de regressão logística foram realizadas para identificar possíveis preditores de relato de sintomas. Há diversas justificativas para o uso desta técnica em particular, mas a principal razão é que permite que sejam investigados os papéis mediadores e moderadores das variáveis intervenientes.

Um conjunto de análises de regressão logística hierárquica foi realizado para cada uma das regiões anatômicas. Variáveis demográficas (idade e gênero) foram incluídas no primeiro bloco, para que se controlasse o seu efeito; o segundo bloco incluiu as variáveis relativas à carga física (tipo de atividade, lotação e as variáveis dummy relativas à carga não ocupacional); variáveis psicossociais (percepção de sobrecarga, suporte social, suporte material ao desempenho, gestão organizacional, política de pagamento e desenvolvimento e estilo de gestão do supervisor) entraram a seguir na equação; o último passo incluiu as variáveis intervenientes (exaustão emocional, coping e hábitos). Finalmente, passos adicionais incluíram módulos interativos para testar possíveis relações indiretas (efeito moderador).

Para tornar mais clara a interpretação dos resultados das análises, os valores de logs odds ou betas foram traduzidos como probabilidade (Hosmer \& Lemeshow, 1989; Pampel, 2000), por meio do cálculo do derivativo parcial (DP),

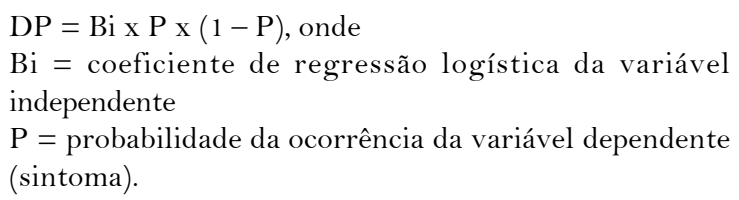

Todas as análises estatísticas foram realizadas por meio do programa SPSS PC+.

\section{Resultados}

Estatísticas descritivas da amostra e a correlação entre as variáveis contínuas do estudo estão descritas na Tabela 1.

A ocorrência de sintomas osteomusculares na amostra pesquisada foi semelhante à exibida em estudos anteriores, com sujeitos empregados em empresas públicas e na iniciativa privada (Pinheiro \& Tróccoli, 2001). Dos participantes, $49 \%$ relataram sintomas em regiões anatômicas periféricas, enquanto $47 \%$ relataram sintomas em regiões anatômicas centrais. Portanto, para o cálculo do $\mathrm{DP}, \mathrm{P}($ sintomas em regiões periféricas $)=0,49$ e $\mathrm{P}($ sintomas em regiões centrais $)=0,47$. A prevalência é alta, levemente superior ao percentual de $41 \%$ demonstrado por Bernard et al. (1994) entre trabalhadores em uma empresa de editoração gráfica.

A Tabela 2 apresenta o resultado do modelo completo de regressão logística hierárquica para sintomas em regiões anatômicas centrais. A importância de preditores individuais 

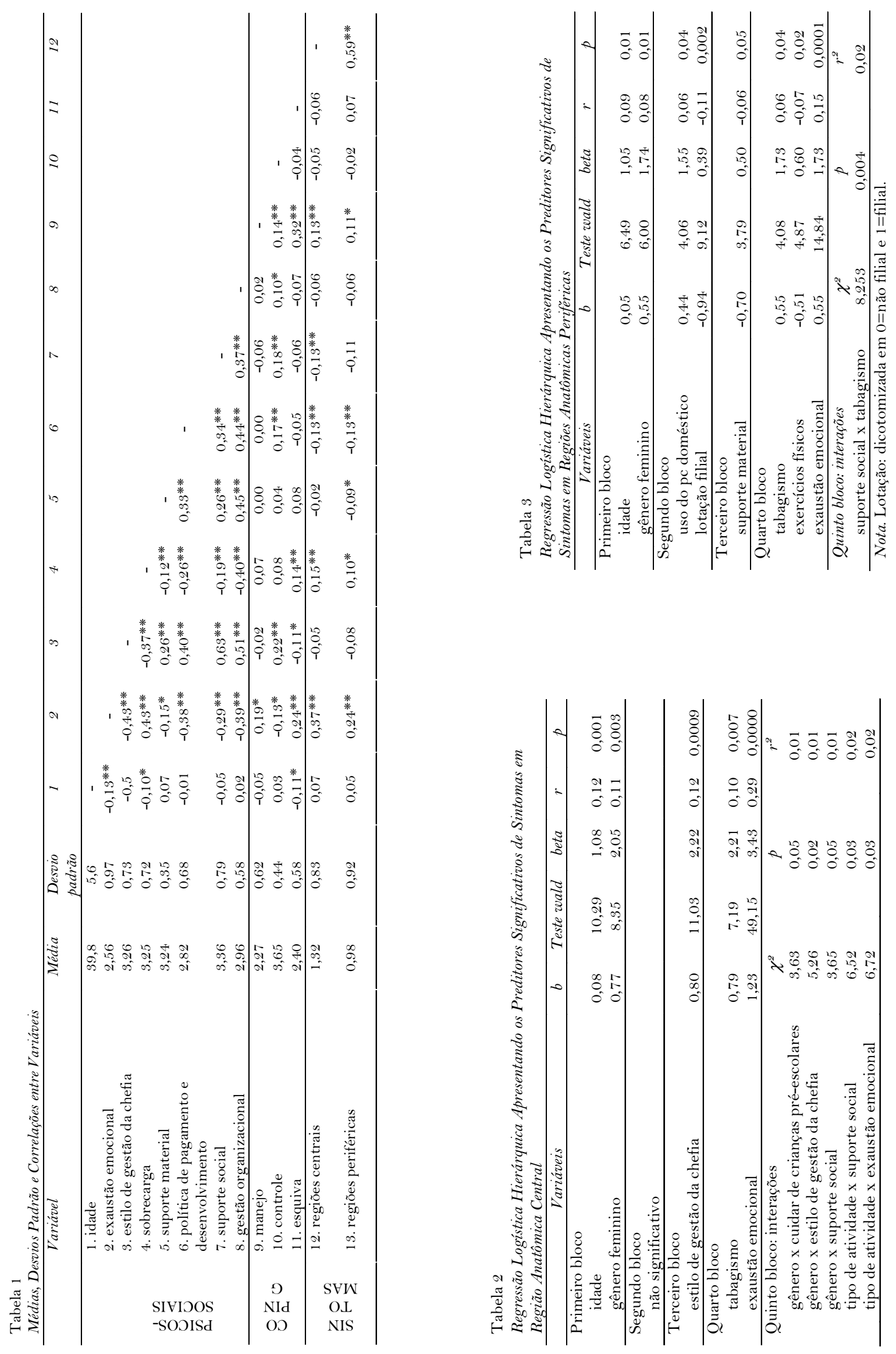
é dada pelos valores de $\mathrm{B}$ (coeficientes de regressão não padronizados), Teste Wald da significância dos coeficientes, $\beta$ (beta, logaritmo da razão odds ou valores de risco relativo), $r$ (correlações parciais) e nível de significância $(p)$. Somente os preditores significativos foram apresentados.

O bloco que incluiu as variáveis demográficas contribuiu para explicar apenas $5 \%$ da variância em sintomas, e apresentou um valor de qui-quadrado=18,30, $p=0,0002$. Idade e gênero foram considerados preditores significativos. O gênero feminino apresentou probabilidade de relatar sintomas 2,05 vezes maior do que o gênero masculino, $p=0,003$. Um aumento de um ano em idade resultou em um aumento da probabilidade de se relatar sintomas da ordem de $2 \%$, conforme cálculo do derivativo parcial, já citado $(d p=0,08 \times 0,47 \times 0,53)$.

$\mathrm{O}$ segundo bloco incluiu as variáveis relativas à carga física. Com um valor de qui-quadrado=14,66 e $p=0,16$, o bloco não foi considerado significativo para explicar variância em sintomas. $\mathrm{O}$ terceiro bloco incluiu as variáveis psicossociais no modelo. O passo apresentou um valor de Qui-quadrado altamente significativo, qui-quadrado=36,24; $p=0,0000$ e acrescentou $12 \%$ à explicação da variância total. A um acréscimo de um ponto na escala de percepção favorável do estilo de gestão da chefia, houve um acréscimo da ordem de $20 \%$ na probabilidade dos participantes relatarem sintomas $(d p=0,80 \times 0,47 \times 0,53)$. Suporte social foi o outro preditor significativo, e a mudança em um ponto na escala, em direção à percepção da existência de uma rede de suporte social, reduziu em $18 \%$ a probabilidade de se relatar sintomas $(d p=-0,75 \times 0,47 \times 0,53)$.

O último passo incluiu as variáveis intervenientes no modelo, com um valor de qui-quadrado $=88,01, p=0,0000 \mathrm{e}$ explicou um percentual de $21 \%$ da variância total. As variáveis responsáveis foram exaustão emocional e tabagismo. Um aumento de um ponto na escala de exaustão emocional aumentou a probabilidade de se relatar sintomas da ordem de $30 \%$ ( $d p=1,23 \times 0,47 \times 0,53)$, enquanto que o hábito de fumar aumentou em $221 \%$ a chance de se relatar sintomas.

O modelo completo explicou 38\% da variância em sintomas nas regiões anatômicas centrais. Módulos interativos foram incluídos ao final da análise para investigar possíveis relações moderadoras, que serão discutidas posteriormente.

A Tabela 3 apresenta os preditores significativos para sintomas em regiões anatômicas periféricas.

O primeiro bloco, que incluiu as variáveis demográficas, explicou $4 \%$ da variância em sintomas e apresentou valor de qui-quadrado=13,86; $p=0,001$. Idade e gênero foram preditores significativos. Mulheres apresentaram probabilidade $74 \%$ maior de relatar sintomas do que homens. A cada ano de idade, a probabilidade de se relatar sintomas aumentou em $1 \%(d p=0,05 \times 0,49 \times 0,51)$.

$\mathrm{O}$ segundo bloco incluiu as variáveis relativas à carga física. Explicando $4 \%$ da variância em sintomas, este bloco apresentou qui-quadrado=12,84 e $p=0,04$. A lotação em Filial pode prevenir o relato de sintomas reduzindo em $61 \%$ a probabilidade do relato para indivíduos lotados naquele subsistema, quando comparados aos empregados lotados em agência e matriz. Carga física não-ocupacional também apresentou poder preditivo em sintomas. $\mathrm{O}$ uso doméstico do microcomputador aumentou em $55 \%$ a probabilidade de indivíduos relatarem sintomas em regiões periféricas.

Dentre as variáveis psicossociais, apenas suporte material foi um preditor significativo, após o ajuste para as outras variáveis do modelo. A mudança em um ponto na escala, denotando percepção favorável do suporte material recebido, diminuiu em $17 \%$ a probabilidade de indivíduos relatarem sintomas $(d p=-0,70 \times 0,49 \times 0,51)$. O bloco apresentou qui-quadrado=24,74; $p=0,0004$ e explicou $6 \%$ da variância total.

O último bloco incluiu as variáveis intervenientes. Com um valor de qui-quadrado=34,06; $p=0,0000$, as variáveis deste bloco contribuíram para explicar $9 \%$ da variância em sintomas. O hábito de fumar aumentou a probabilidade do relato de sintomas em $73 \%$, enquanto que a prática de exercícios físicos reduziu em $40 \%$ a probabilidade dos sujeitos relatarem sintomas. $\mathrm{O}$ aumento em um ponto na escala de exaustão emocional esteve associado ao aumento percentual no relato de sintomas da ordem de $14 \%$ ( $d p=0,55$ x $0,49 \times 0,51)$.

O modelo completo explicou 23\% da variância em sintomas nas regiões anatômicas periféricas.

\section{Discussão}

De forma geral, o modelo de relações proposto foi confirmado pelos resultados. Fatores relativos à carga física e ao ambiente psicossocial foram preditores independentes de sintomas nas duas grandes regiões anatômicas, confirmando os resultados de estudos como os de Bernard (1997) e o de Toomingas et al. (1997). Carga física decorrente da lotação em diferentes subsistemas da empresa teve efeito direto sobre o relato de sintomas em regiões anatômicas periféricas, e tal efeito não foi verificado sobre o relato de sintomas em regiões anatômicas centrais, onde sua influência foi indireta; variáveis psicossociais puderam prever mais fortemente sintomas em regiões centrais, e foram fracos preditores de sintomas em regiões periféricas, onde também exerceram influência indireta, moderada por hábitos. A conclusão é que nenhuma das duas grandes regiões anatômicas é refratária à influência de carga física ou das variáveis psicossociais, para esta amostra de trabalhadores em ambiente de escritório. Diferentes configurações dos fatores etiológicos devem ser esperadas em atividades que incluam a utilização de força física.

O quinto bloco da Tabela 2 mostra que gênero moderou a relação entre o cuidar de crianças em idade pré-escolar e o relato de sintomas (a relação foi significativa para homens e não para mulheres). Exaustão emocional foi também moderadora da relação entre tipo de atividade e sintomas em regiões centrais. Observa-se que altos escores em exaustão emocional só foram relacionados ao aumento no relato de sintomas quando a atividade envolvia alta freqüência de digitação. 
Observou-se ainda o papel moderador de suporte social na relação entre tipo de atividade e sintomas. O efeito de reduzir a probabilidade do relato de sintomas só foi observado para atividades que não envolvem grande carga física. Para aquelas atividades em que a digitação é freqüente, o aumento na escala de percepção de suporte não esteve associado a decréscimos significativos na média de sintomas. Estes resultados apresentam a importância da combinação de fatores relativos à carga física e psicossocial na predição da morbidade osteomuscular e representam a contribuição deste trabalho, à medida que sugerem outros mecanismos de associação entre as variáveis além dos efeitos diretos.

Há fracas evidências da importância de carga física de natureza não-ocupacional na morbidade osteomuscular, pelo menos na forma em que foi mensurada neste estudo. As variáveis apresentaram importância marginal como preditores de sintomas em ambas as regiões anatômicas. Observou-se que as atividades que oferecem risco nãoocupacional são de tipos diferentes para homens e mulheres, o que pode ocasionar baixas freqüências esperadas em algumas celas e comprometer o poder da análise de regressão logística (Tabachnick \& Fidell, 1996). Estudos posteriores deverão considerar atividades não profissionais que sejam igualmente exercidas por homens e mulheres, a fim de clarificar o seu papel relativo.

Confirmando os resultados de diversos estudos, gênero mostrou-se um fator importante na predição de sintomas em ambas as regiões anatômicas. Carga física decorrente da atividade ocupacional ou de fontes não ocupacionais, bem como o nível de reatividade ao estresse não são as únicas explicações para este fato. Williams e Wiebe (1999) sugeriram que uma das possíveis razões pelas quais as mulheres relatam mais sintomas é que elas experimentariam mais estresse do que os homens, mas esta explicação não é coerente com os resultados aqui apresentados. Mulheres apresentaram médias em exaustão emocional significativamente superiores aos homens (2,65 e 2,45, respectivamente, $p=0,03)$, assim como algumas diferenças significativas relativamente às variáveis psicossociais e a utilização das estratégias de coping. Entretanto, gênero permaneceu um preditor significativo mesmo após o controle destas variáveis. Aquelas autoras hipotetizaram ainda que diferenças no processamento cognitivo de informações relativas à saúde poderiam resultar em que homens e mulheres utilizariam estratégias de coping diferentes, ocasionando maior freqüência de relato entre as mulheres. De fato, se o relato de sintomas está associado à busca de suporte, ele pode ser mais provável entre as mulheres do que entre os homens. Esta possibilidade de associação entre gênero e relato de sintomas é coerente com o que propôs Koslowsky (1998). Aquele autor também assinalou a importância dos fatores culturais na explicação da maior vulnerabilidade feminina ao estresse.

Efeitos indiretos de gênero na relação entre variáveis do ambiente ocupacional e morbidade osteomuscular em regiões centrais foram também encontrados. A relação entre a percepção do estilo de gestão e o relato de sintomas foi invertida para homens e mulheres: enquanto homens foram menos propensos a relatar sintomas quando percebiam o supervisor de forma muito apoiadora ou muito pouco apoiadora, entre as mulheres, deu-se o contrário. Elas tenderam a relatar mais sintomas em situações em que perceberam chefias muito apoiadoras ou muito pouco apoiadoras. Este resultado, novamente, aponta para diferenças no estilo de coping de homens e mulheres, particularmente quanto às estratégias de buscar auxílio.

Os resultados não apoiaram a hipótese segundo a qual as mulheres tenderiam a relatar mais sintomas em razão da maior carga física de natureza não-ocupacional, principalmente pela execução de atividades domésticas e pela responsabilidade por cuidar de crianças pequenas. Considerando que carga física, para esta amostra de sujeitos exercendo atividades de escritório, não foi um fator primordial para explicar variância em sintomas, é bem possível que a carga adicional, decorrente das atividades domésticas, também não seja relevante. Além disso, as mulheres que compuseram a amostra têm jornada de trabalho média de oito horas diárias, o que só se torna viável se o trabalho doméstico, ou parte dele, houver sido delegado a uma outra pessoa.

Variáveis psicossociais tiveram mais importância na explicação de sintomas em regiões anatômicas centrais. Com exceção das variáveis percepção da gestão organizacional do desempenho e da política de pagamento e desenvolvimento, as demais foram preditores significativos de morbidade osteomuscular.

Suporte social mostrou efeito direto, reduzindo a probabilidade do relato de sintomas em regiões centrais, além do efeito indireto ou buffering(House, 1981), em reforço aos resultados de Bongers et al. (1993), Bergqvist et al. (1995), Hales et al. (1994), Ahlberg-Hulten, Theorell, e Sigala (1995).

O efeito indireto de suporte social foi verificado na relação entre tabagismo e sintomas em regiões periféricas. $\mathrm{O}$ hábito foi um preditor significativo, que aumentou em cerca de $70 \%$ a probabilidade do relato de sintomas naquela região anatômica. Entretanto, entre os tabagistas, a média de sintomas diminuiu à medida em que eles relataram uma percepção mais favorável de suporte social. Dado que não há indícios de que a percepção de suporte social seja maior entre tabagistas do que entre os não tabagistas, carga física pode explicar este resultado. Uma possível razão seria as pausas freqüentes que os tabagistas realizam durante o expediente, freqüentemente em grupo, o que poderia aliviar a carga física de forma a impedir traumas cumulativos decorrentes da atividade ininterrupta.

A percepção do estilo do supervisor mostrou um efeito direto sobre sintomas em regiões centrais particularmente interessante. Independentemente de sua fraca correlação bivariada negativa com o relato de sintomas $(r=-0,07)$, foi um preditor significativo para morbidade osteomuscular, como um fator de risco. Este resultado aponta para o fenômeno descrito por Cohen e Cohen (1975, pp.89-91) e 
por Tabachnick, e Fidell (1996, p.165) como supressão. Uma variável independente que teria o poder de predizer fracamente o efeito tem a sua importância exacerbada no contexto da análise de regressão em razão da supressão da variância irrelevante. $\mathrm{O}$ fenômeno é confirmado pelos sinais invertidos do índice de correlação bivariada e do coeficiente de regressão não padronizado da variável independente em relação à variável critério. Neste caso, os resultados da variável estilo de gestão, no último passo da análise de regressão são considerados espúrios. De qualquer forma, as análises parciais do modelo mostraram que suporte social foi mediador da relação entre a percepção do estilo do supervisor e morbidade osteomuscular.

Percepção de sobrecarga foi também um preditor de sintomas em regiões centrais, mas seu efeito foi completamente mediado por exaustão emocional, o que vale dizer que não houve contribuição adicional da variável além daquela atribuída à medida de reatividade ao estresse ocupacional. Este último resultado é um indício de que o estresse é uma das vias pelas quais o ambiente psicossocial exerce seu impacto sobre a saúde das estruturas osteomusculares.

A percepção quanto ao estilo de gestão organizacional e à política de pagamento e desenvolvimento da empresa não foram capazes de prever sintomas em regiões centrais ou periféricas. Suporte material apresentou efeito direto sobre sintomas em regiões periféricas, mesmo após o controle de carga física e de exaustão emocional. A variável, como um fator de suporte organizacional ao desempenho, parece representar, para os empregados, o interesse da empresa para com a sua saúde e bem-estar.

Exaustão foi, além do mais poderoso preditor de sintomas em ambas as regiões, um mediador perfeito da relação entre percepção de sobrecarga e sintomas em regiões anatômicas centrais. $\mathrm{O}$ efeito mediador de exaustão não foi verificado para outras variáveis psicossociais. A variável foi ainda moderadora da relação entre carga física e sintomas em regiões centrais.

Nenhuma das variáveis de coping foi preditor direto de sintomas em qualquer das regiões anatômicas. A análise de relações interativas mostrou que para indivíduos lotados em agência, onde as atividades repetitivas são mais comuns, a maior utilização de estratégias de esquiva está associada à redução do relato de sintomas, o que não se verificou para as demais lotações. Isto aponta para a importância do caráter situacional na avaliação da eficácia das estratégias de coping no ambiente ocupacional. Possivelmente, apenas este grupo de estratégias esteja disponível aos indivíduos para lidar com os estressores típicos daquela situação. Outros estudos poderão desenvolver medidas de coping que contemplem a especificidade do ambiente ocupacional de forma a abranger maior amplitude de estratégias do que o instrumento aqui utilizado.

É necessário alertar para algumas limitações deste trabalho. Além dos viezes relativos aos estudos transversais, a taxa de respostas de $28 \%$ pode não ser considerada suficiente para evitar o viés oriundo da seleção da amostra
(Dickinson et al., 1992). A medida de carga física eliminou o viés do auto-registro, mas pode envolver outros vieses não considerados a princípio, como a possibilidade de haver divergências importantes entre as atividades constantes da descrição do cargo e as efetivamente desempenhadas pelos sujeitos. Finalmente, o poderoso efeito de exaustão emocional sugere cautela na interpretação dos resultados. Outras pesquisas poderão usar técnicas de modelo de equação estrutural para estimar possíveis relações de reciprocidade.

\section{Referências}

Ahlberg-Hulten, G. Theorell, T., \& Sigala, F. (1995). Social support, job strain and musculoskeletal pain among female health care personnel. Scandinavian Journal of Work, Environment and Health, 21, 435-439.

Bernard, B. P. (Ed.) (1997). Work-related musculoskeletal disorders and psychosocial factors. Cincinati, OH: NIOSH, National Institute for Occupational Safety and Health.

Bernard, B., Sauter, S., Fine, L., Petersen, M., \& Hales, T. (1994). Job task and psychosocial risk factors for work-related musculoskeletal disorders among newspaper employees. Scandinavian Journal of Work, Environment and Health, 20, 417-426.

Bergqvist V., Wolgast, E., Nilsson, B., \& Voos, M. (1995). The influence of VDT work on musculoskeletal disorders. Ergonomics, 38, 754-762.

Bongers, P.M., de Winter, C.R., Kompier, M.A.Jr, \& Hildebrandt, V.H. (1993). Psychosocial factors at work and musculoskeletal disease. Scandinavian Journal of Work, Environment and Health, 19, 297-312.

Cohen J. \& Cohen, P. (1975). Applied Multiple Regression: Correlation Analysis for the Behavioral Sciences. New York: Erlbaum.

Dickinson, C.E., Campion, K., Foster, A.F., Newman, S.J., O’Rourke, A.M.T., \& Thomas, P.G. (1992). Questionnaire development: an examination of the Nordic Musculoskeletal Questionnaire. Applied Ergonomics, 23, 197-205.

Elovainio M. \& Sinervo, T. (1997). Psychosocial stressors at work, psychological stress and musculoskeletal symptoms in the care for the elderly. Work and Stress, 11, 351-361.

Hales, T.R., Sauter, S.L., Peterson, M.R., Fine, L.J., PutzAnderson, V., Schleifer, L.R., Ochs, T.T., \& Bernard, B.P. (1994). Musculoskeletal disorders among visual display terminal users in a telecommunications company. Ergonomics, 37, 1603-162 1.

Hosmer, D.W.Jr. \& Lemeshow, S. (1989). Applied Logistic Regression. New York: John Wiley \& Sons.

House, J.S. (1981). Work stress and social support. Massachusetts: Addison-Wesley.

Houtman, I., Bongers, P., Smulders, P., \& Kompier, M. (1994). Psychosocial stressors at work and musculoskeletal problems. Scandinavian Journal of Work, Environment and Health, 20, 139-145.

Koslowsky, M. (1998). Modelling the stress-strain relationship in work settings. London: Routledge.

Kuorinka, I., Jonsson, B., Kilbom, A., Vinterberg, H., BieringSorensen, F., Andersson, G., \& Jorgensen, K. (1987). Standardised Nordic questionnaires for the analysis of musculoskeletal symptoms. Applied Ergonomics, 18, 233-237.

La Rocco, J.M., House, J.S., \& French, J.R.P. (1980). Social support, occupational stress and health. Journal of Health and Social Behavior, 21, 202-218. 
Latack, J.C. (1986). Coping with job stress: measures and future directions for scale development. Journal of Applied Psychology, 71, 377-385.

Leino, P. \& Haenninem, V. (1995). Psychosocial factors at work in relation to back and limb disorders. Scandinavian Journal of Work, Environment and Health, 21, 134-142.

Lima, M.E.A. (1997). A LER no setor bancário.Cidade: Health.

Miranda, C.F. (1998). Introdução à saúde no trabalho. São Paulo: Atheneu.

Moon, S.D. (1996). A psychosocial view of cumulative trauma disorders: implications for occupational health and prevention. In S.D. Moon \& S.L. Sauter (Eds.), Beyond biomechanics: psychosocial aspects of musculoskeletal disorders in office work. London: Taylor \& Francis.

Pampel, F.C. (2000). Logistic regression: a primer. London: Sage.

Parkes, K.R. (1999). Shiftwork, job type, and the work environment as joint predictors of health-related outcomes. Journal of Occupational Health Psychology, 4, 256-268.

Parkes, K.R., Mendham, C.A., \& von Rabenau, C. (1994). Social support and the demand-discretion model of job stress: tests of additive and interactive effects in two samples. Journal of Vocational Behavior, 44, 91-113.

Pinheiro, F.A. \& Tróccoli, B.T. (2001). Além dos fatores físicos: preditores psicossociais das doenças osteomusculares relacionadas ao trabalho [Resumo]. In Resumos de Comunicações Científicas (cd-rom), II Congresso Norte Nordeste de Psicologia, Salvador, BA.

Pinheiro, F.A., Tróccoli, B.T., \& Carvalho, C.V. (2002). Questionário Nórdico de Sintomas Osteomusculares: validade do instrumento como medida de morbidade. Revista de Saúde Pública, 36, 307-312.

Ribeiro, H.P. (1997). Lesões por esforços repetitivos (LER): uma doença emblemática. Cadernos de Saúde Pública, 13, 85-93.

Rio, R.P. (1998). LER: Ciência e Lei. Belo Horizonte: Health.

Sato, L., Araújo, M.D., Udihara, M.L., Franco, M.A., Nicotera, F.N., Daldon, M.T., Settimi, M.M., \& Silvestre, M.P. (1993). Atividade em grupo com portadores de L.E.R. e achados sobre a dimensão psicossocial. Revista Brasileira de Saúde Ocupacional, 79(2 1), 49-62.

Sauter, S., Schleifer, L.M., \& Knutson, S.J. (1991). Work posture, workstation design and musculoskeletal discomfort in a VDT data entry task. Human Factors, 33, 151-167.
Selye, H. (1985). History and present status of the stress concept. In A. Monat \& R.S. Lazarus (1985). Stress and Coping: an Anthology. New York: Columbia University Press.

Smith, M.K., \& Carayon, P. (1996). Work organization, stress, and cumulative trauma disorders. In S.D. Moon \& S.L Sauter (Eds.), Beyond biomechanics: psychosocial aspects of musculoskeletal disorders in office work. London: Taylor \& Francis.

Tabachnick, B.G. \& Fidell, L.S. (1996). Using multivariate statistics. New York: Harper Collins.

Tamayo, M.R., \& Tróccoli, B.T.(2000). Construção e validação de uma versão modificada da subescala de exaustão emocional: reinterpretando o construto de burnout [Resumos]. In Anais da $52^{a}$ Reunião Anual da SBPC, Brasília, DF.

Theorell, T. (1996). Possible mechanisms behind the relationship between the demand-control-support model and disorders of the locomotor system. In S.D. Moon \& S.L. Sauter (Eds.), Beyond biomechanics: psychosocial aspects of musculoskeletal disorders in office work. London: Taylor \& Francis.

Toomingas, A., Theorell, T., Michelsen, H., \& Nordemar, R. (1997). Associations between self-rated and psychosocial work conditions and musculoskeletal symptoms and signs. Scandinavian Journal of Work, Environment and Health, 23, 130-139.

Westgaard, R.H. (1996). Effects of psychological demand and stress on neuromuscular function. In S.D. Moon \& S.L. Sauter (Eds.), Beyond biomechanics: psychosocial aspects of musculoskeletal disorders in office work. London: Taylor \& Francis.

Williams, P.G. \& Wiebe, D.J. (2000). Individual differences in selfassessed health: gender, neuroticism and physical symptom reports. Personality and Individual Differences, 28, 823-835.
Submissão: 25/08/2004 $1^{a}$ revisão: $11 / 02 / 2005$ Última revisão: 20/10/2005 Aceite final: 08/11/2005 